C. Aerts, T.R. Bedding, \& J. Christensen-Dalsgaard, eds.

\title{
Pulsations of Complete Evolutionary Models for roAp Stars: Preliminary Results
}

\author{
G. Michaud ${ }^{1}$, P. Brassard, J. Richer, O. Richard ${ }^{1}$, G. Fontaine \\ Département de physique, Université de Montréal, Canada, H3C $3 J^{\gamma}$
}

\section{Pulsation code}

The evolutionary models described in the accompanying poster (Richard et al., these proceedings) have been tested for pulsations using both the adiabatic code developed by Brassard et al. (1992) and the corresponding non-adiabatic code as first used in Fontaine et al. (1994). For the stars of interest, these codes have the great advantages of being potentially able to treat atmospheric regions, as well as being numerically stable and efficient. The required derivatives were calculated by least-square fitting of spline functions to complete opacity tables constructed at each composition of the evolved model. Since there were some 1500 zones for each model, 1500 such tables were constructed and spline functions were fitted to each of them. They then gave directly the derivatives required for the pulsation calculations (see Fig. 1). Note that models equivalent to $1.70 \mathrm{~T} 5.3 \mathrm{D} 1 \mathrm{~K}-3$ have already been tested for pulsations by Turcotte et al. (2000).

\section{Adiabatic properties, excitation and damping}

If one plots the Brunt-Väisälä frequencies (not shown) as a function of $\log q$ $(q \equiv \Delta M / M)$ for the three models, the main differences between the 1.70r1M-3 model and the others appear close to the surface. They can be related to the $B$ term which, as discussed in Brassard et al. (1991) - see their Eq. (15), refers to rapid variations of the mean molecular weight. It suggests a possibility that thin layers of heavy elements close to the surface would play a role in the pulsation properties. A large difference at $\log q=-6.5$ is caused by the Fe accumulation and the corresponding $\mathrm{Fe}$ convection zone.

We have compared work integrals for some modes to determine if atomic diffusion modifies chemical composition sufficiently to affect pulsation properties. Does one of the assumed turbulence distributions lead to increased excitation of the modes involved in roAp stars?

Our models span a range of properties. In particular, two of them have large underabundances of $\mathrm{He}$ and a $\mathrm{Fe}$ abundance peak at $\log q=-6.5$ leading to a peak in opacity and a convection zone (see Richard et al., these proceedings). Differential work integrals for the $\ell=1, n=10$ gravity mode of the three $1.7 M_{\odot}$ models are shown on Fig. 1. This mode has a period of $\sim 36700 \mathrm{~s}$. There is driving of the mode in the region of the maximum in the opacity caused by

${ }^{1}$ CERCA, 5160 boul. Décarie, Montréal, PQ, Canada, H3X 2H9 

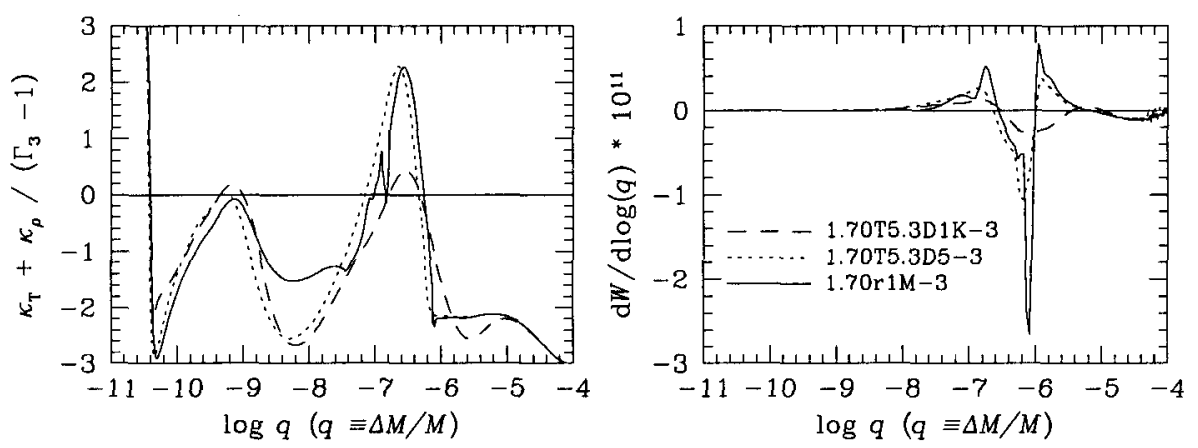

Figure 1. Left panel: derivatives of the opacity. Right panel: differential work integral for the $n=10, \ell=1$, g-mode.

the Fe peak (at $\log q \sim-6.5$ ). There also appears to be weaker driving deeper in, caused by the broad opacity peak from $\mathrm{Fe}$ in the region where its radiative acceleration is larger than gravity (at $\log q \sim-4.5$ ). In between the two, there is damping apparently related to the minimum in the Fe concentration. The mode is stable, however, implying that the damping outside those regions dominates.

\section{Conclusion}

In this study, we did not include any effect of the magnetic field on convective properties. The preliminary results are that the increased damping caused by the reduction of He concentration (in the 1.70r1M-3 and 1.70T5.3D5-3 compared to the $1.70 \mathrm{~T} 5.3 \mathrm{D} 1 \mathrm{~K}-3$ model) is compensated, for some modes, by the increased driving caused by metals. We did not find any excited mode. However, according to Balmforth et al. (2001) at the end of their Sect. 5.2, the main reason for the pulsations they obtain is that they suppress, because of the magnetic field, the damping caused by turbulent pressure fluctuations in convective zones. Since we do not include such an effect, one should not expect the increased driving by iron peak elements in our calculations to lead to pulsations, even if it replaces completely that lost because of reduced He.

\section{References}

Balmforth, N.J., Cunha, M.S., Dolez, N., Gough, D.O., \& Vauclair, S. 2001, MNRAS, 323, 362

Brassard, P., Fontaine, G., Wesemael, F., Kawaler, S.D., \& Tassoul, M. 1991, ApJ, 367, 601

Brassard, P., Pelletier, C., Fontaine, G., \& Wesemael, F. 1992, ApJS, 80, 725

Fontaine, G., Brassard, P., Wesemael, F., \& Tassoul, M. 1994, ApJ, 428, L61

Turcotte, S., Richer, J., Michaud, G., \& Christensen-Dalsgaard, J. 2000, A\&A, 360,603 